\title{
Study on the Instability of Two-Phase Flow in the Heat-Absorbing Tube of Trough Solar Collector
}

\author{
Ying Zhang, ${ }^{1}$ Peiyao Liu, ${ }^{1}$ Peisheng Li, ${ }^{1,2}$ Yue Chen, ${ }^{1}$ and Yanni Pan ${ }^{1}$ \\ ${ }^{1}$ School of Mechanical and Electrical Engineering, Nanchang University, Nanchang, Jiangxi 330031, China \\ ${ }^{2}$ Shangrao Normal College, Shangrao, Jiangxi 334001, China \\ Correspondence should be addressed to Peisheng Li; ncudns1995z@163.com
}

Received 26 July 2017; Accepted 30 August 2017; Published 23 October 2017

Academic Editor: Ben Xu

Copyright (c) 2017 Ying Zhang et al. This is an open access article distributed under the Creative Commons Attribution License, which permits unrestricted use, distribution, and reproduction in any medium, provided the original work is properly cited.

\begin{abstract}
The Marangoni effect and Rayleigh-Benard effect in the two-phase region of solar trough heat-absorbing tube are simulated by FTM (front tracking method). Considering the Marangoni effect alone, although surface tension gradient and surface tension affect the interface wave, the two effects have different characteristics. The surface tension gradient caused by the temperature gradient is one of the factors that swing the interface. The amplitude attenuation of the interface wave decreases with the increase of the Marangoni number (Ma). In general, the surface tension gradient enhances the convection opposite to the temperature gradient. Under the gravity field, the Rayleigh-Benard effect influences the development of the vortex structure in the flow field, which in turn affects the velocity gradient near the interface to influence the evolution of the interface fluctuation. In a small Rayleigh number $(\mathrm{Ra})$, the buoyancy convection reduces the velocity gradient, thus suppressing the evolution of the interfacial wave. In the range of $\mathrm{Ra}<4.0 E 4$, the larger the $\mathrm{Ra}$, the stronger the inhibitory effect. However, when the Ra number is large $(\mathrm{Ra}>4.0 E 4)$, the situation is just the opposite. The larger the $\mathrm{Ra}$ is, the stronger the promoting effect is.
\end{abstract}

\section{Introduction}

Heat-absorbing tube, the primary part of the solar thermal system, is a device used to convert solar energy to heat energy. Its stability and heat transfer efficiency inside have a direct impact on the operation of the whole system. A lot of researchers have studied its aspects. By numerical calculation, Muñoz and Abánades [1] and Kumar and Reddy [2] obtained results showing that changing the geometrical parameters can enhance the heat transfer and reduce the temperature gradient in the tube like adding a porous disk and using helical internal fins, thereby reducing heat loss. Flores and Almanza [3] designed a copper-steel composite bimetallic heat pipe which is helpful to reduce the circumferential temperature difference. Agrafiotis et al. [4] pointed out that the use of good thermal shock resistance of the ceramic material can effectively overcome the damage caused by temperature gradient. It is obvious to find that the temperature gradient inside is always harmful, which is too easy to cause the pipe bending, heat loss increase, and a series of problems. However, scholars are more concentrated on the pipe material, structure or other external factors, and the effect of the radial temperature gradient on the flow in the tube, and the resulting heat loss had few analyses.

Poor radial temperature distributions create Marangoni and Rayleigh-Benard effects. The Marangoni effect and the Rayleigh-Benard effect cover large areas [5-7], included covering slags, contaminant transport, hypermonotectic alloy etc. Two effects were discovered very early $[8,9]$, but in recent years, scholars paid close attention to it again because this seemingly insignificant perturbation has a significant impact on fluid flow instability, heat transfer, and mass transfer [10-12]. The study for the two effects is mainly focused on the causes of convection and the effect of heat transfer and mass transfer on convections [13, 14]; however, little attention has been paid to the change of interface motion caused by the convective process.

As mentioned above, the Marangoni effect and RayleighBenard effect in heat-absorbing tube have been studied 
extensively, but the influence of the two effects on the interface fluctuation has not been explored thoroughly, due to the high-precision demand for interface tracking. Therefore, in order to study the interfacial fluctuation under the effect of convection caused by temperature gradient, this paper adopts a numerical method (FTM) with high interfacial numerical accuracy $[15,16]$, a numerical simulation of interface fluctuation within a two-phase region in a heatabsorbing tube is carried out, and some characteristics of Marangoni and Rayleigh-Benard effects on the variation of the interfacial fluctuation are found, which are expected to improve the internal working environment and to develop higher efficiency heat-absorption.

\section{Mathematical Model and Numerical Method}

\subsection{Phase-Interface Tracking N-S Equation and Energy Equation}

Assumptions. The fluid is incompressible Newtonian fluid, and the fluid density change satisfied the Boussinesq approximation without heat source; its two-dimensional flow momentum equation and energy equation can be written as follows:

$$
\begin{aligned}
\rho \frac{\partial u}{\partial t}+\rho \nabla \cdot u u & =-\nabla p+\rho g \beta\left(T-T_{L}\right)+\mu \nabla^{2} u+f, \\
\frac{\partial \rho c_{p} T}{\partial t}+\nabla \cdot\left(\rho c_{p} T u\right) & =\nabla \cdot(k \nabla T) .
\end{aligned}
$$

These equations are valid for the whole flow field, and the various fluids can be identified by a step (Heaviside) function $H$, which is written in Section 2.4, where $u$ is velocity, $p$ is pressure, $\rho$ is the fluid density, $\mu$ is viscosity, $g$ is acceleration of gravity, $k$ is the thermal conductivity, $c_{p}$ is the heat capacity at constant pressure, $T$ is the temperature, $f$ is the volumetric forces at the interface regardless of gravity, $\beta$ is the thermal expansion coefficient, and $T_{\mathrm{L}}$ is the reference temperature in the Boussinesq model, which is the temperature of the cold wall.

2.2. The Surface Tension. Using the CSF (continuous-surfaceforce) method, the expression is

$$
F_{\sigma}=\int_{s}\left[\sigma(T) k n+\frac{\partial \sigma(T)}{\partial(s)} t\right] \delta\left(x-x^{\mathrm{f}}\right) d s,
$$

where $\delta\left(x-x^{\mathrm{f}}\right)$ is a Dirac function that when on the interface shows 1 and if not shows 0 .

The surface tension is assumed to vary linearly with temperature, and the expression is as follows:

$$
\sigma(T)=\sigma_{0}+\gamma\left(T-T^{*}\right)
$$

where $\gamma$ is the temperature coefficient, in general, which has a negative value, $T^{*}$ is the initial average temperature of the interface, and the temperature of the interface point is obtained from the fixed grid by bilinear interpolation.
2.3. Moving the Interface. The interface is usually combined with a fixed grid; the exchange of information between the interface and the grid is achieved through the area weight function. After the bilinear interpolation is performed, the moving speed of the interface can be obtained (well, the interface is always moving without the need of calculating the speed). Using a simple first-order explicit time integral, we can get the interface position in the next time step.

$$
x_{f}^{(n+1)}=x_{f}^{n}+\Delta t u_{f}^{n}
$$

where $x_{f}^{(n+1)}$ and $x_{f}^{n}$ are the position of the interface at time $n+1$ and $n$, respectively, $\Delta t$ is the time step, and $u_{f}^{n}$ is the velocity of the interface at time $n$.

2.4. Reconstruction of Physical Field. For immiscible and incompressible fluid, fluid holds its characteristics, respectively, at both sides of the interface. When the interface moves, the distribution of interfacial parameter also changes, so we use the Heaviside function to characterize this change:

$$
\mathrm{H}(\varphi)=\left\{\begin{array}{l}
1, \quad \varphi>\alpha \\
\frac{1}{2}\left(1+\frac{\varphi}{\alpha}+\frac{1}{\pi}\left(\frac{\pi \varphi}{\alpha}\right)\right), \quad\|\varphi\| \leq \alpha \\
0, \quad \varphi<-\alpha,
\end{array}\right.
$$

where $\varphi$ is the distance to the interface of a given point and $2 \alpha$ is the thickness of the transition region between the absolute value of the two fluids. Therefore, the distribution of density, viscosity, thermal conductivity, and constant pressure heat capacity is obtained by using the abovementioned instruction function.

2.5. The Physical Model. As shown in Figure 1(a), the heatabsorbing tubes are placed horizontally and there are three sections. The second zone of the steam-water two-phase zone is taken as the research object as shown in Figure 1(b). $T_{\mathrm{H}}$ and $T_{\mathrm{L}}$ are the radiation surface temperature (high temperature) and the back radiation surface temperature (low temperature), respectively. The temperature difference of the high and low temperature wall is set to 100; all the physical quantities used in this article have been nondimensionlized. The calculated domain size is $L \times L=1 \times 1$, the grid number is $600 \times 600$, where $h$ is the grid spacing, $\Delta t=0.06 h$, and an initial disturbance of 0.1 at $Y=0.5$ was provided, due to the existence of relative movement between the upper and lower layers of fluid; therefore, an initial shear field [17] can be set as follows:

$$
1(x, y)=\tan h\left[\frac{y-0.5-0.01 \sin (2 p x)}{0.02}\right] .
$$

The fluid's initial speed is defined as follows:

$$
\begin{aligned}
& u(x, y, 0)=\theta l(x, y), \\
& v(x, y, 0)=0,
\end{aligned}
$$

where $\theta$ is the velocity coefficient. 


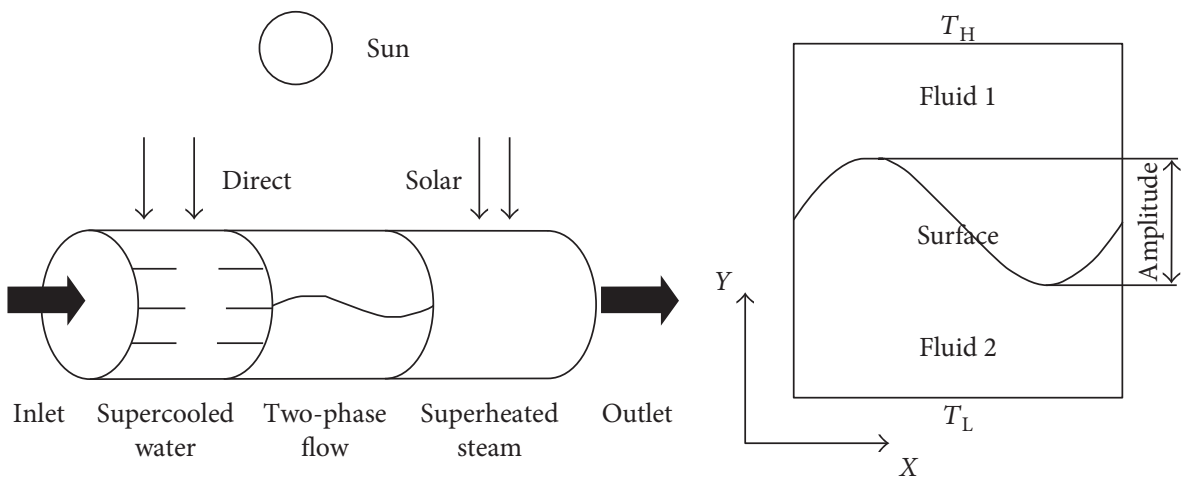

(a) Schematic diagram of the heat-absorbing tube

(b) Simplify the model

Figure 1: Physical model.

2.6. Model Verification. Where there is large shear force across an initial amplitude between two-component fluids, the interface is unstable, which is called Kelvin-Helmholtz instability. According to the results from Lee and Kim [17]. When we set $h=1 / 256, \Delta t=0.064 h, \operatorname{Re}=5000$, and $g=\sigma$ $=0$, the analytical nondimensional growth rate $\gamma_{\mathrm{e}}$ can be written as follows:

$$
\gamma_{\mathrm{e}}=\frac{4 \pi \sqrt{r}}{1+r}
$$

$r$ is the density ratio between two fluids.

The numerical growth rate $\gamma_{\mathrm{n}}$ can be written as

$$
\gamma_{\mathrm{n}}=\frac{A(t) /\left(A_{0}-1\right)}{t}
$$

where $A_{0}$ is the initial amplitude.

In this paper, a model verification was provided by comparing with the numerical results from analytical results as shown in Figure 2. It depicts that two results are in good agreement. The conclusion shows that the numerical method of this paper can realize the description of the interface instability process well.

2.7. Dimensionless Definition. The Marangoni number was defined as

$$
\mathrm{Ma}=\frac{\gamma|\nabla T| d}{\mu_{2} a_{2}} .
$$

The Reynolds number was defined as

$$
\operatorname{Re}=\frac{\rho_{2} u_{0} d}{\mu_{2}}
$$

where $u_{0}$ is the initial velocity of the fluid in the gravity field defined as $u_{0}=g \rho_{2} d^{2} / 2 \mu_{2}$.

The Rayleigh number was defined as

$$
\mathrm{Ra}=\frac{\beta \rho_{2} g \nabla T d^{3}}{\mu_{2} a_{2}} .
$$

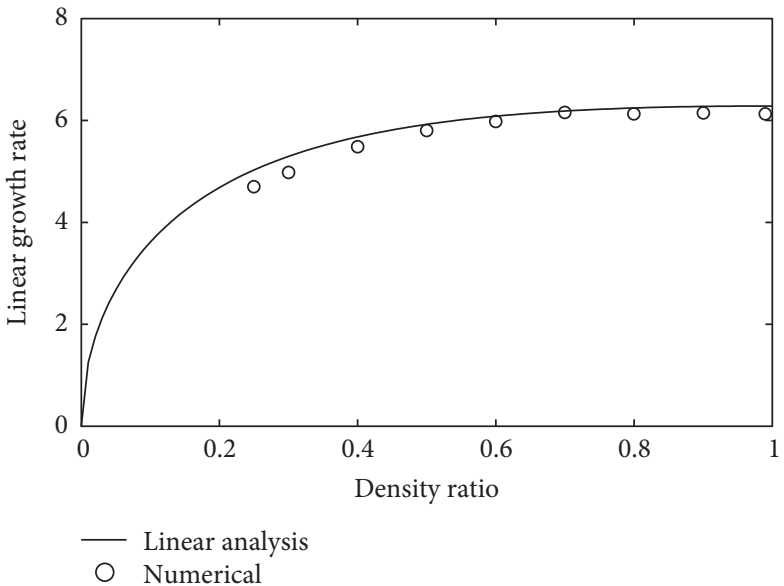

FIgURE 2: Comparison of simulation results with analytical results.

\section{Result and Discussion}

3.1. Marangoni Effect on Liquid Membrane Flow. In microgravity or zero gravity, the Rayleigh-Benard effect is very weak; in order to highlight the effects of the Marangoni effect, gravity is not considered here. Considering that the velocity of the two-phase zone in the heat-absorbing tube is small, when the Reynolds number is small, the fluid instability will not continue to develop but will be restored due to the smaller shear force at the interface. At the same time, due to the smaller size of convective heat transfer in the flow field, a vertical downward temperature gradient can still be formed near the interface. There will be two effects at this point; on the one hand, as the temperature changes, the surface tension coefficient accordingly. As shown in Figures 3(a), 3(b), and $3(\mathrm{c})$, in the initial stage $(t=0.03)$, the pressure produced by the interface varies obviously due to the different coefficients of surface tension. The larger the Ma number is, the lower the pressure difference at the wave crest (high temperature) is, and the greater the pressure difference at the trough (low temperature) is. In addition, it can be found in Figure 3(a) that the internal and external pressures are approximately symmetrical, but Figures 3(b) and 3(c), Ma is not zero. Due to the effect of the tangential surface tension, the internal 


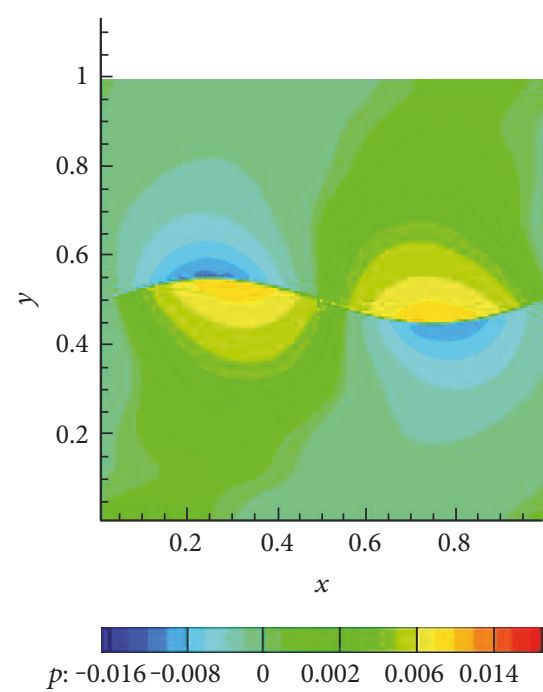

(a) $\mathrm{Ma}=0$

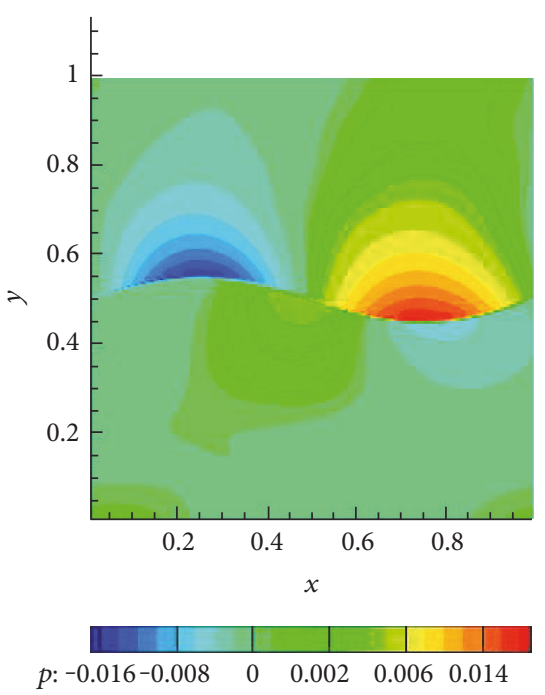

(b) $\mathrm{Ma}=3.3 E 4$

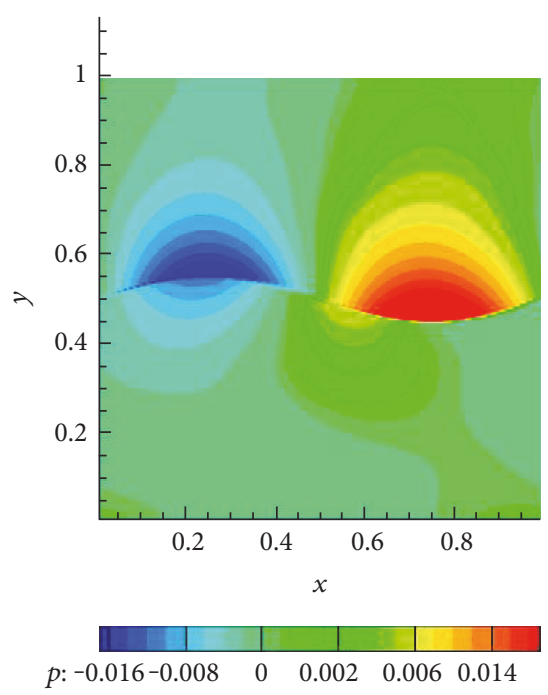

(c) $\mathrm{Ma}=6.6 E 4$

Figure 3: The pressure distribution of the interface at $T=0.03$ time, $\operatorname{Re}=12.5$.

and external pressures at the crest and trough interface become asymmetrical.

On the other hand, the surface tension gradient caused by the temperature gradient affects the motion of the interface, which inhibits perturbations moving in the same direction of the temperature gradient and promotes perturbations moving in the opposite direction of the temperature gradient. The numerical value of the surface tension affects the normal surface tension, while the surface tension gradient affects the tangential surface tension. In order to distinguish the difference between the normal surface tension and the tangential surface tension, Figure 4 depicts the time dependent curves of the peaks and troughs of the interface when the coefficient of surface tension and the number of $\mathrm{Ma}$ are changed, respectively. As can be seen from the figure, the wave crests and troughs are symmetrically changed under the same conditions. In further analysis, comparing the curves of $\mathrm{Ma}=0$, sigma (surface tension coefficient) $=0.01$, and $\mathrm{Ma}=0$, sigma $=0.02$ in Figure 4, increasing the surface tension coefficient reduces the fluctuation period, and when the number of fluctuations is the same, the fluctuation range of the crest and trough increases. But comparing the curves of sigma $=0.01, \mathrm{Ma}=0$ and sigma $=0.01, \mathrm{Ma}=3.33 E 4$, at the crest of the latter, the surface tension decreases due to the influence of temperature, and only from this point of view, the wave period of crest should be increased. However, the reaction in the curve is not the case because the surface tension gradient is downward, and the period of wave crest decreases at the trough; the surface tension coefficient is greater than the average because the temperature is lower; if only the increase of the coefficient of surface tension is considered, the magnitude of the trough recovery should be increased. Because of the downward surface tension gradient, the upward recovery movement is suppressed, and the upper pole of the first movement of $\mathrm{Ma}=3.33 E 4$ is smaller than that of $\mathrm{Ma}=0$; comparing the curves of sigma $=0.01, \mathrm{Ma}=1.33 E 4$ and sigma $=0.01, \mathrm{Ma}=3.33 E 4$, respectively, from the two sets of curves, we can see that the difference of peaks and troughs due to the surface tension gradient increases with the increase in the Ma numbers. At the later stage of the motion, the disturbance amplitude is gradually smaller, and the repeated fluctuation make the single temperature gradient near the interface gradually disappear, and the disturbance fluctuation gradually becomes symmetrical. On the whole, in the Marangoni system, the existence of the surface tension gradient increases the unstable factors, and the larger the $\mathrm{Ma}$ number is, the longer is the time required for the interface disturbance to recover.

Because of the gradient of surface tension, the flow field is strengthened in the direction of temperature gradient and the temperature change near the bottom of the interface is affected. This change fluctuates with the fluctuation of the interface, and the fluctuation is similar. Figure 5 shows the change of the average temperature between 0.2 and 0.4 in the longitudinal direction of the flow field ( $y$-axis). When the number of $\mathrm{Ma}$ is zero, the bottom temperature changes with a very symmetrical periodic change, while the number of $\mathrm{Ma}$ is not equal to zero. The temperature fluctuation amplitude is greatly enhanced. At the beginning of the movement, as the deformation of the interface begins to recover, the bottom temperature begins to decrease, but the surface tension gradient promotes downward convection; the larger the Ma number is, the smaller the temperature drop is and the faster the temperature rise is. In addition, the increase of the Ma number increases the magnitude of the temperature rise.

\subsection{The Effect of Rayleigh-Benard Effect on the Stability of} Fluid Interface under Gravity. In the normal gravity field, the Marangoni effect is almost negligible compared with the Rayleigh-Benard effect. Therefore, the influence of the Rayleigh-Benard effect on the flow field of the fluid interface is studied by setting the upper fluid in the opposite direction to the underlying fluid; the influence of the Marangoni effect 


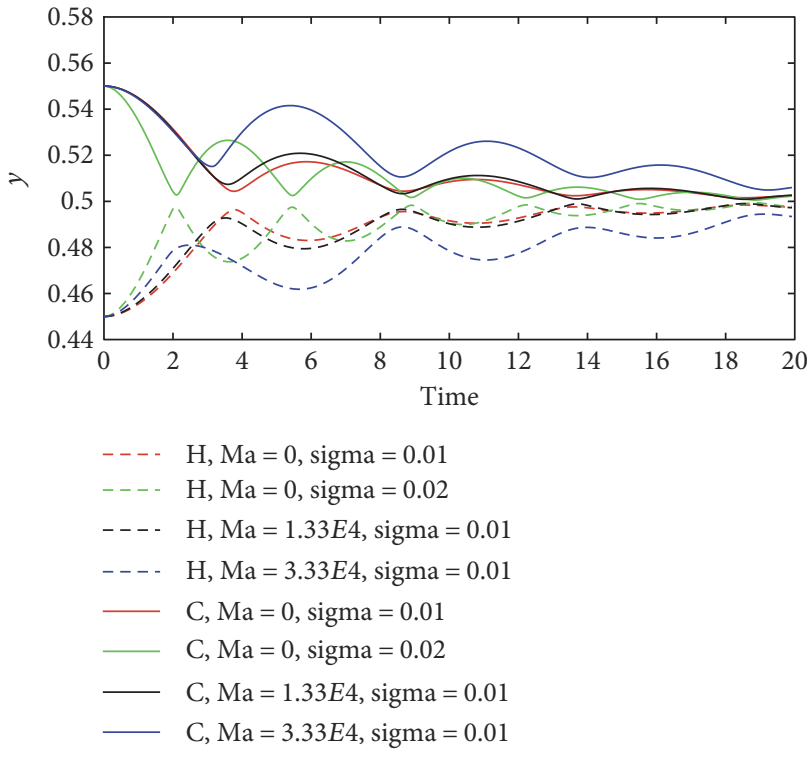

Figure 4: Curve of the position of wave crest and trough with time. $\mathrm{H}$ is the trough, $\mathrm{C}$ is the crest, $\mathrm{Re}=12.5$.

is ignored. Figure 6 shows the streamline with $\mathrm{Ra}=0,1.25 E 4$, and $6.25 E 4$ and with $T=0.09,1.99$, and 4.44 , respectively. In order to observe it better, a separate color note was used each time. As shown in Figure 6, the vortices generated by the Rayleigh-Benard effect interacts with the original vortices. When the $\mathrm{Ra}$ number is small $(\mathrm{Ra}=1.25 E 4$, Figures $5(\mathrm{~b})$, $5(\mathrm{e})$, and $5(\mathrm{~h})$ ), the center of the flow field generates a vortex with a larger vortex velocity. When the Ra number is large $(\mathrm{Ra}=6.25 E 4$, Figures 5(c), 5(f), and 5(i)), the eddy current in the center of the flow field does not change significantly in the $\mathrm{X}$ direction, but the velocity of turbulence increases obviously on both sides. When $t=1.99$ and $\mathrm{Ra}=0$, the vortex develops only near the interface, and in the case of $\mathrm{Ra}=1.25 E 4$, the range of the vortex becomes larger and is almost filled with the half of area. And two plume vorticities are produced in the lower left corner and the lower right corner. The kinetic energy is constantly dissipated in the rotary motion, so the velocity of the fluid decreases gradually. In the case of $\mathrm{Ra}=6.25 E 4$, the distribution of vortices is similar to that of $\mathrm{Ra}=0$, only at the vicinity of the interface, and the velocity of the upper fluid becomes larger. By the influence of the Rayleigh-Benard effect, when $\mathrm{Ra}$ is not zero, the flow rate of the lower fluid decreases. Moreover, the speed at $\mathrm{Ra}=1.25 E 4$ decreases by a greater magnitude than that of $\mathrm{Ra}=6.25 E 4$. The flow rate of the upper fluid is different from that of the lower fluid. At $\mathrm{Ra}=1.25 E 4$, the flow rate of the upper fluid decreases, while when $\mathrm{Ra}=6.25 E 4$, the flow rate of the upper fluid increases significantly. In general, when $\mathrm{Ra}=1.25 E 4$, the difference of velocity between fluids decreases; when $\mathrm{Ra}=6.25 E 4$, it suggests the opposite. Another big difference is the vortex structure in later stage, when $\mathrm{Ra}=0$, it almost disappears; in the case when $\mathrm{Ra}$ is not zero, there is always a vortex at the center of the flow field.

Figure 7 shows the time-dependent curves of amplitude in different $\mathrm{Ra}$ number. At $\mathrm{Ra}=0$, the velocity gradient

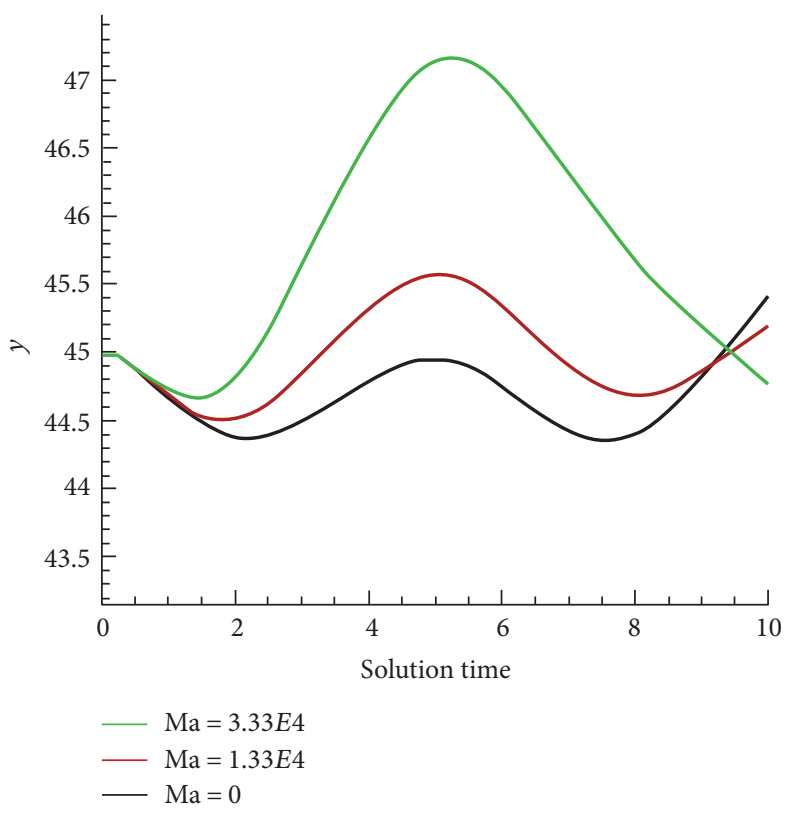

FIGURE 5: Temperature changes of bottom.

between fluids is so large that the surface tension can no longer overcome the shear force and maintain the original state, which results in the appearance of the fingers at the interface; this phenomenon has been mentioned by [18]. When $\mathrm{Ra}=1.25 E 4$, the Rayleigh-Benard effect reduces the velocity difference, and the disturbance does not produce the fingerlike protrusions, but the amplitude of interface decreases gradually. When $\mathrm{Ra}=6.25 E 4$, the difference increases and the amplitude increases. In addition, the interface development does not monotonically because of the existence of vortices, and by the same reason, tremors becomes unstable after $T=5$ in the case of $\mathrm{Ra}=6.25 E 4$ and $\mathrm{Ra}=0$.

So the influence of the Rayleigh-Benard effect on the interface perturbation is found to have two opposites effects when the Ra number is small and the $\mathrm{Ra}$ number is large. As shown in Figure 7, since the phase of the interface wave is different in different cases, the second trough of the curve in Figure 7 is taken as a form. It can be seen from Figure 8 that in the range of $1.0 E 4<\mathrm{Ra}<4.00 E 4$, the amplitude of the interface wave decreases with the increase of Ra number, that is, with the increase of $\mathrm{Ra}$ number, the inhibition of buoyancy convection on the interfacial wave instability is increasingly strong, which is opposite to the case of $4.0 E 4<\mathrm{Ra}<6 E .04$.

\section{Conclusions}

In this paper, we consider the flow effect caused by the uneven temperature distribution in the basic FTM model and simulate the evolution process of the interface wave at the shear field with or without gravity, providing a theoretical basis for the optimization design of the heat-absorbing tube. The specific research results are as follows:

(1) In the shear field, when only the Marangoni effect is considered, the interface evolution is affected by 


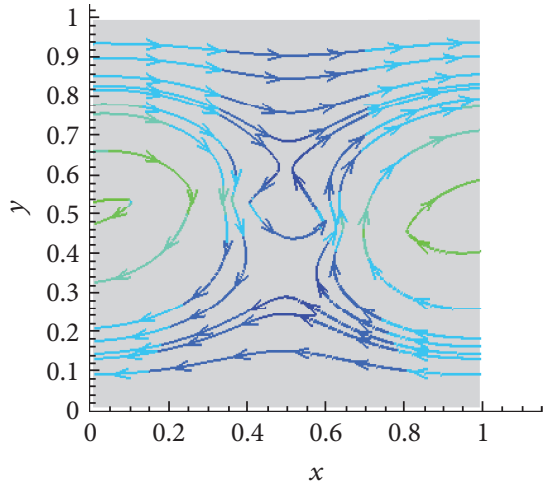

Velocity magnitude:

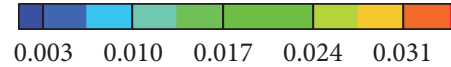

(a) $\mathrm{Ra}=0, t=0.09$

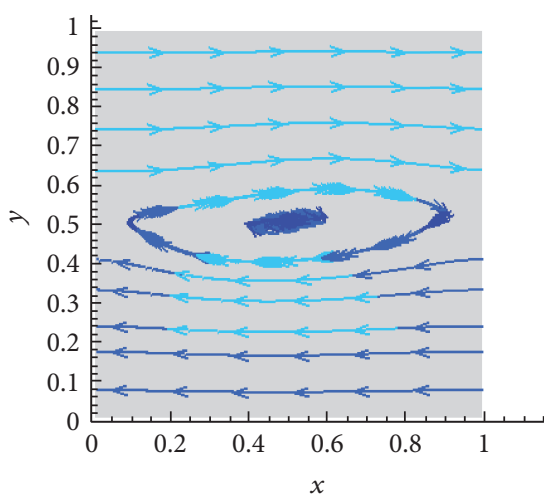

Velocity magnitude:

\begin{tabular}{|cc|c|c|c||}
\hline & & & & $\mid$ \\
0.092 & 0.275 & 0.458 & 0.642 & 0.825
\end{tabular}

(d) $\mathrm{Ra}=0, t=1.99$

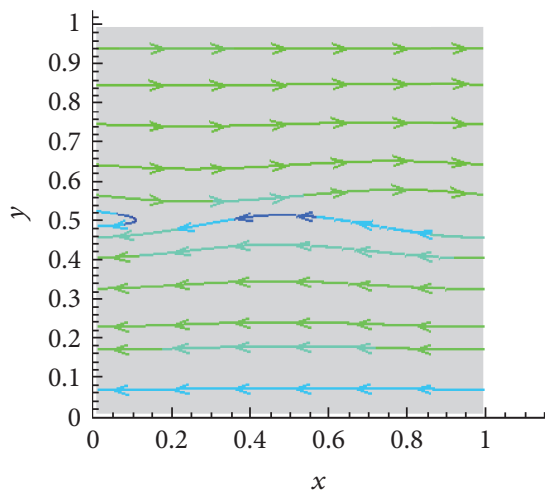

Velocity magnitude:

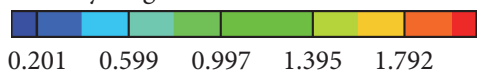

(g) $\mathrm{Ra}=0, t=4.44$

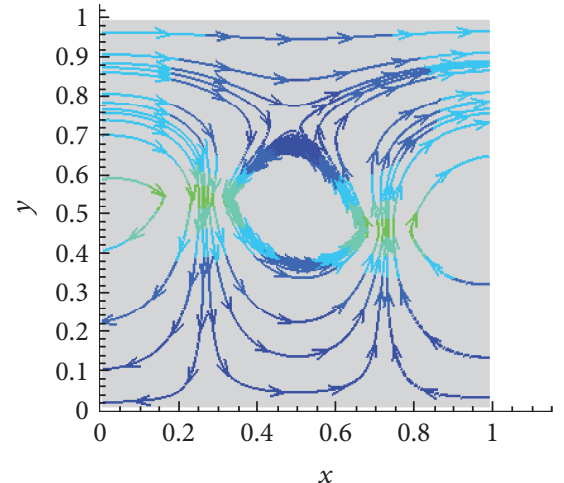

Velocity magnitude:

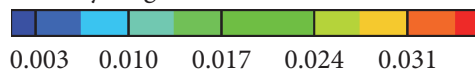

(b) $\mathrm{Ra}=1.25 E 4, t=0.09$

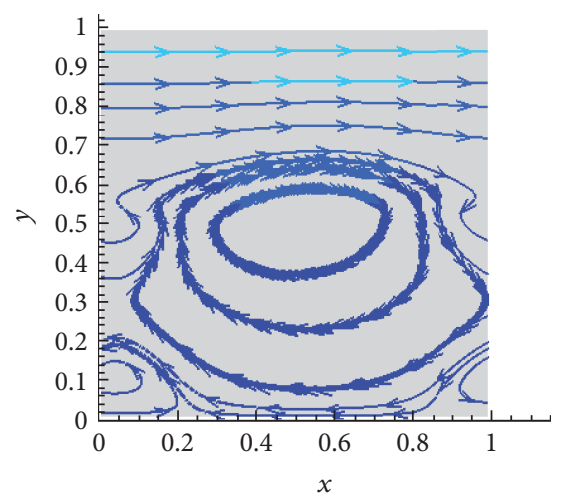

Velocity magnitude:

\begin{tabular}{|cc|c|cc|c|c}
\hline & & & & & \\
\hline 0.092 & 0.275 & 0.458 & 0.642 & 0.825
\end{tabular}

(e) $\mathrm{Ra}=1.25 E 4, t=1.99$

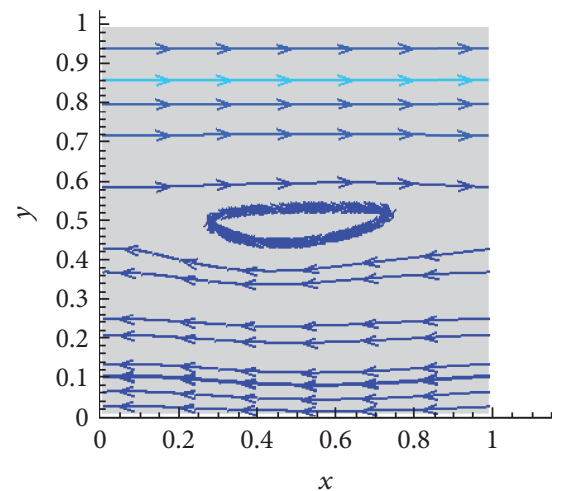

Velocity magnitude:

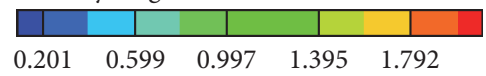

(h) $\mathrm{Ra}=1.25 E 4, t=4.44$

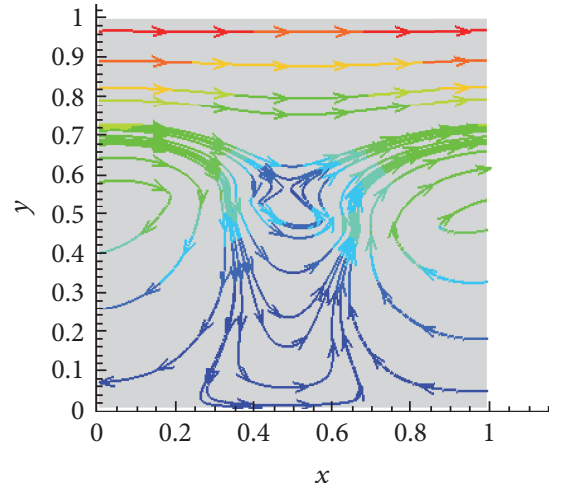

Velocity magnitude:

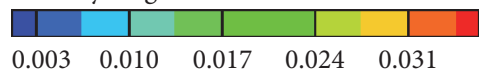

(c) $\mathrm{Ra}=6.25 E 4, t=0.09$

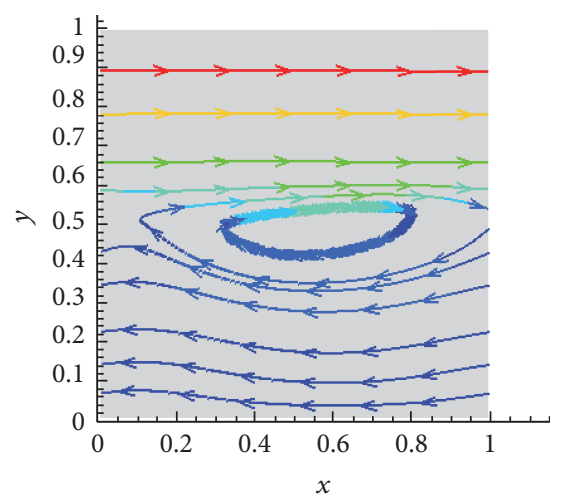

Velocity magnitude:

\begin{tabular}{llllll|l}
\hline & & & & & \\
\hline 0.092 & 0.275 & 0.458 & 0.642 & 0.825
\end{tabular}

(f) $\mathrm{Ra}=6.25 E 4, t=1.99$

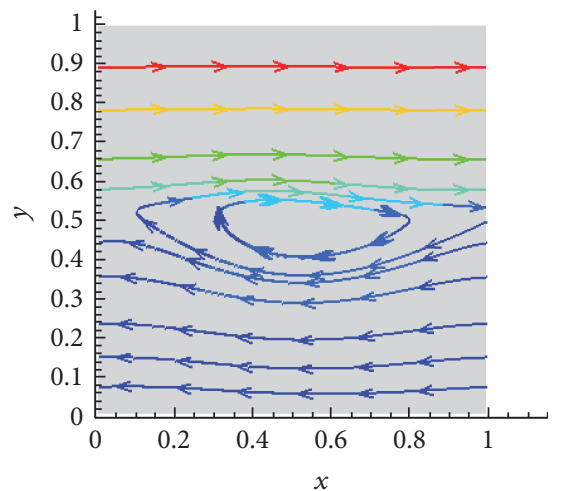

Velocity magnitude:

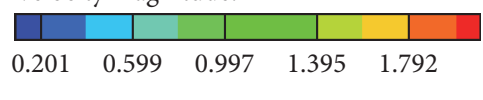

(i) $\mathrm{Ra}=6.25 E 4, t=4.44$

Figure 6: Streamlines in different Ra numbers.

both surface tension and the gradient of surface tension. When the motion direction of the interface is the same as that of the surface tension gradient, the motion is promoted. As a whole, the surface tension gradient increases the instability due to the temperature change, and the attenuation of the amplitude becomes slower. Therefore, the choice of large temperature coefficient of the 


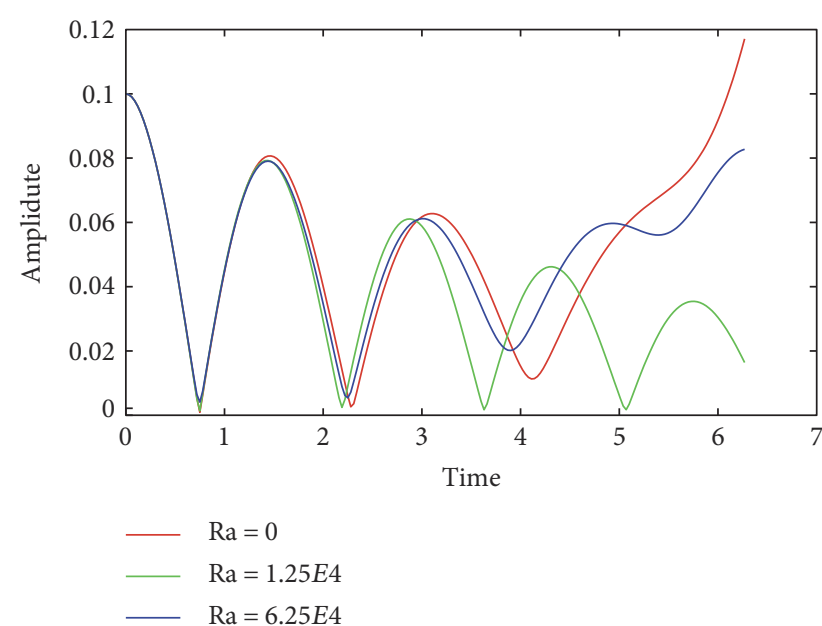

Figure 7: Curves of interface amplitude versus time when $\mathrm{Ra}=0$, $1.25 E 4,6.25 E 4$.

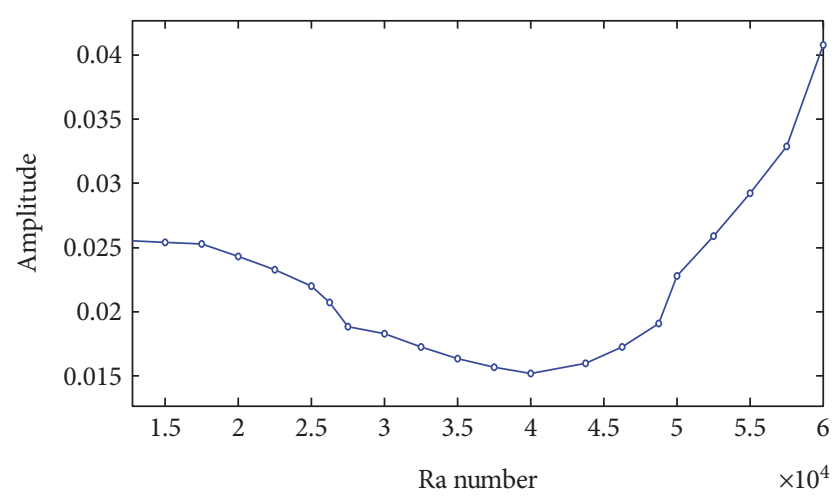

FIGURE 8: Disturbance amplitude in different Ra number when at $T=6$ times.

fluid can enhance the heat transfer in the heatabsorbing tube.

(2) In the gravitational field, the Marangoni effect and the Rayleigh-Benard effect work together, but compared with the Marangoni effect, the RayleighBenard effect dominates and does not have obvious directionality; at first, it affects the development of the flow field vortex structure, and then the change of the velocity gradient near the interface affects the evolution of the interfacial fluctuations. When the Ra number is small, the buoyancy convection causes the velocity gradient to decrease and suppress the evolution of the interface wave, and the function is stronger in no other $\mathrm{Ra}$ number than 4.0E4. However, when the Ra number is large, the situation is exactly the opposite to the small one. From what has been discussed above, it is better to let the Ra number stay away from $4.0 E 4$ by some means such as the fluid's density, viscosity and thermal expansion coefficient, which make the flow field vortex movement most violently, and is the most favorable for heat transfer.

\section{Conflicts of Interest}

The authors declare that there is no conflict of interest regarding the publication of this paper.

\section{Acknowledgments}

The authors gratefully acknowledge the support under the National Natural Science Foundation of China (Grant no. 11562011) and the funds from Hundred Sail Away Project in 2015 provided by Jiangxi Provincial Party Committee Organization Department and Jiangxi Association for Science and Technology.

\section{References}

[1] J. Muñoz and A. Abánades, "A technical note on application of internally finned tubes in solar parabolic trough absorber pipes," Solar Energy, vol. 85, no. 3, pp. 609-612, 2011.

[2] K. R. Kumar and K. S. Reddy, "Thermal analysis of solar parabolic trough with porous disc receiver," Applied Energy, vol. 86, no. 9, pp. 1804-1812, 2009.

[3] V. Flores and R. Almanza, "Behavior of the compound wall copper-steel receiver with stratified two-phase flow regimen in transient states when solar irradiance is arriving on one side of receiver," Solar Energy, vol. 76, no. 1-3, pp. 195198, 2004.

[4] C. C. Agrafiotis, I. Mavroidis, A. G. Konstandopoulos et al., "Evaluation of porous silicon carbide monolithic honeycombs as volumetric receivers/collectors of concentrated solar radiation," Solar Energy Materials and Solar Cells, vol. 91, no. 6, pp. 474-488, 2007.

[5] Y. Ueshima, "Evaluation of local dissolution rates and wetting behaviors of solid alumina in liquid slag with the Marangoni number," ISIJ International, vol. 56, no. 8, pp. 1506-1508, 2016.

[6] P. Lakshmanan and P. Ehrhard, "Marangoni effects caused by contaminants adsorbed on bubble surfaces," Journal of Fluid Mechanics, vol. 647, no. 9, pp. 143-161, 2010.

[7] H. B. Cui, C. Y. Li, K. F. Wang, G. F. Mi, J. J. Guo, and Y. Q. Su, "Effect of Marangoni convection on microstructure evolution of $\mathrm{Cu}-\mathrm{Pb}$ hypermonotectic alloy by phase field simulation," Foundry, vol. 57, no. 11, pp. 1176-1180, 2008.

[8] K. Mukai, "Recent studies on the interfacial phenomena in which Marangoni effect participates," Tetsu-to-Hagané, vol. 71, no. 11, pp. 1435-1440, 1985.

[9] E. Bodenschatz, W. Pesch, and G. Ahlers, "Recent developments in Rayleigh-Bénard convection," Annual Review of Fluid Mechanics, vol. 32, no. 1, pp. 709-778, 2000.

[10] Z. Alloui and P. Vasseur, "Onset of Marangoni convection and multiple solutions in a power-law fluid layer under a zero gravity environment," International Journal of Heat and Mass Transfer, vol. 58, no. 1-2, pp. 43-52, 2013.

[11] T. P. Lyubimova, D. V. Lyubimov, and Y. N. Parshakova, "Implications of the Marangoni effect on the onset of Rayleigh-Benard convection in a two-layer system with a deformable interface," The European Physical Journal Special Topics, vol. 224, no. 2, pp. 249-259, 2015.

[12] L. V. Matveev, "Impurity transport in developed RayleighBénard convection," International Journal of Heat and Mass Transfer, vol. 95, pp. 15-21, 2016. 
[13] P. G. Siddheshwar, V. Ramachandramurthy, and D. Uma, "Rayleigh-Bénard and Marangoni magnetoconvection in Newtonian liquid with thermorheological effects," International Journal of Engineering Science, vol. 49, no. 10, pp. 1078-1094, 2011.

[14] A. B. Pieri, F. Falasca, J. von Hardenberg, and A. Provenzale, "Plume dynamics in rotating Rayleigh-Bénard convection," Physics Letters A, vol. 380, no. 14, pp. 1363-1367, 2016.

[15] W. Tauber, S. O. Unverdi, and G. Tryggvason, "The nonlinear behavior of a sheared immiscible fluid interface," Physics of Fluids, vol. 14, no. 8, pp. 2871-2885, 2002.

[16] J. Zhang, D. M. Eckmann, and P. S. Ayyaswamy, “A front tracking method for a deformable intravascular bubble in a tube with soluble surfactant transport," Journal of Computational Physics, vol. 214, no. 1, pp. 366-396, 2006.

[17] H. G. Lee and J. Kim, "Two-dimensional Kelvin-Helmholtz instabilities of multi-component fluids," European Journal of Mechanics - B/Fluids, vol. 49, pp. 77-88, 2015.

[18] X. M. Ye, Y. Zhang, S. L. Wang, and C. X. Li, "Investigation on stability of sheared wavy liquid films," Proceedings of the Chinese Society of Electrical Engineering, vol. 27, no. 20, pp. 103-106, 2007. 

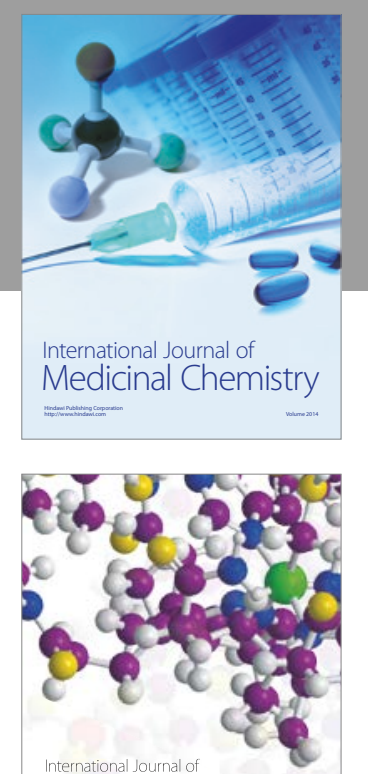

Carbohydrate Chemistry

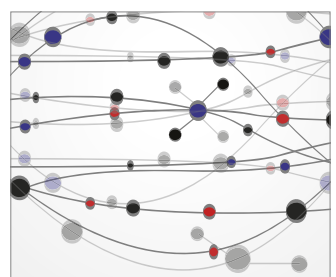

The Scientific World Journal
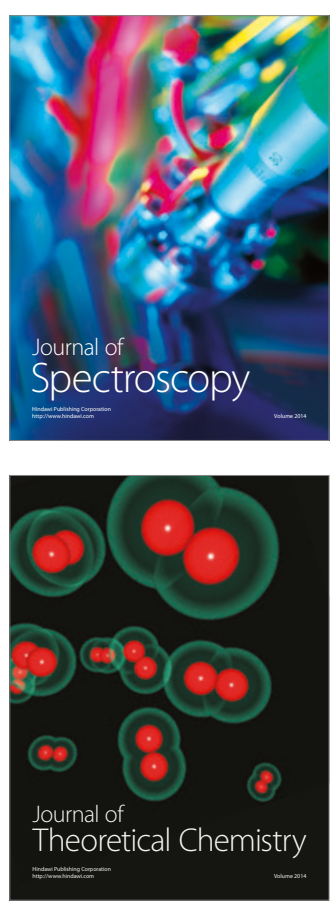
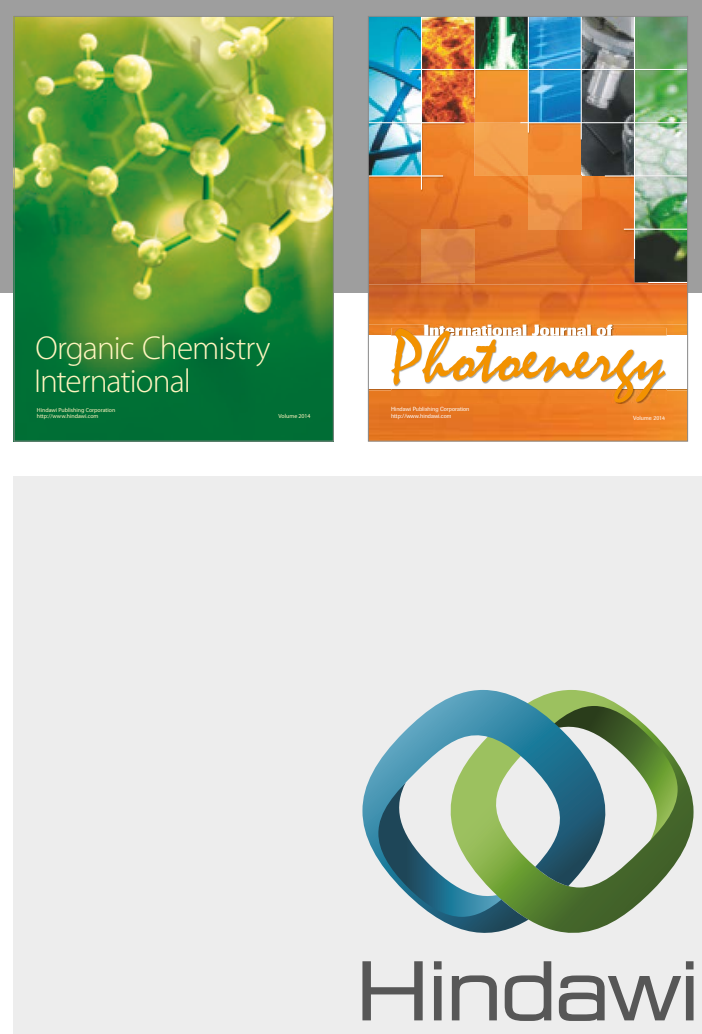

Submit your manuscripts at

https://www.hindawi.com

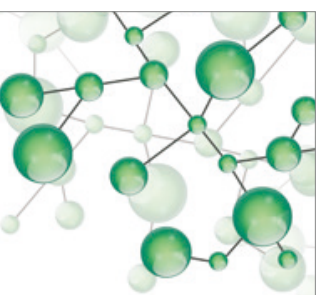

International Journal of

Inorganic Chemistry

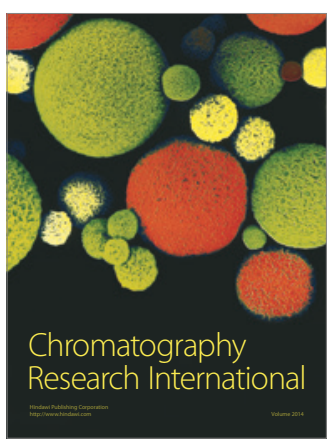

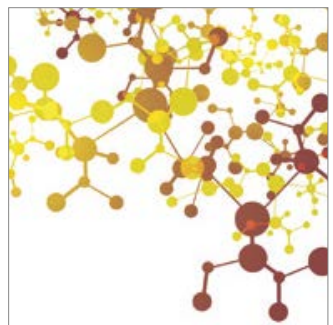

Applied Chemistry
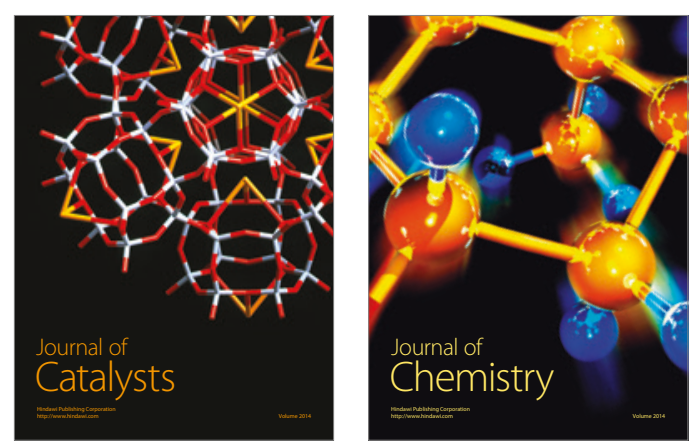
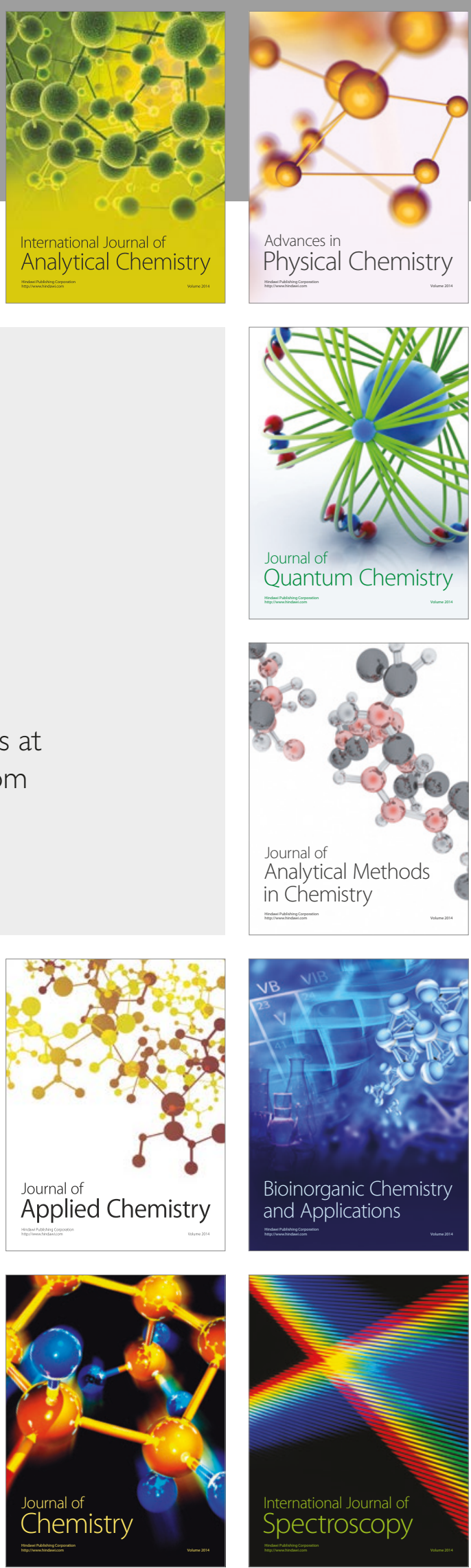